\title{
Icariin potentiates the antitumor activity of gemcitabine in gallbladder cancer by suppressing NF-KB
}

Dian-cai ZHANG, Jin-long LIU, Yong-bin DING, Jian-guo XIA*, Guo-yu CHEN*

Department of General Surgery, The First Affiliated Hospital, Nanjing Medical University, Nanjing 210029, China

\begin{abstract}
Aim: Gemcitabine has been increasingly prescribed for the treatment of gallbladder cancer. However, the response rate is low. The aim of this study is to determine whether icariin, a flavonoid isolated from Epimedi herba, could potentiate the antitumor activity of gemcitabine in gallbladder cancer.

Methods: Human gallbladder carcinoma cell lines GBC-SD and SGC-996 were tested. Cell proliferation and apoptosis were analyzed using MTT assay and flow cytometry, respectively. The expression of apoptosis- and proliferation-related molecules was detected with Western blotting. Caspase-3 activity was analyzed using colorimetric assay, and NF-KB activity was measured with ELISA. A gallbladder cancer xenograft model was established in female BALB/c (nu/nu) mice. The mice were intraperitoneally administered gemcitabine $(125 \mathrm{mg} / \mathrm{kg})$ in combination with icariin $(40 \mathrm{mg} / \mathrm{kg})$ for 2 weeks.

Results: Icariin (40-160 $\mathrm{gg} / \mathrm{mL}$ ) dose-dependently suppressed cell proliferation and induced apoptosis in both GBC-SD and SGC-996 cells, with SGC-996 cells being less sensitive to the drug. Icariin $(40 \mu \mathrm{g} / \mathrm{mL})$ significantly enhanced the antitumor activity of gemcitabine $(0.5 \mu \mathrm{mol} / \mathrm{L})$ in both GBC-SD and SGC-996 cells. The mice bearing gallbladder cancer xenograft treated with gemcitabine in combination with icariin exhibited significantly smaller tumor size than the mice treated with either drug alone. In GBC-SD cells, icariin significantly inhibited both the constitutive and gemcitabine-induced NF-kB activity, enhanced caspase-3 activity, induced $\mathrm{G}_{0}-\mathrm{G}_{1}$ phase arrest, and suppressed the expression of $\mathrm{Bcl}-2, \mathrm{Bcl}-\mathrm{xL}$ and surviving proteins.

Conclusion: Icariin, by suppressing NF-kB activity, exerts antitumor activity, and potentiates the antitumor activity of gemcitabine in gallbladder cancer. Combined administration of gemcitabine and icariin may offer a better therapeutic option for the patients with gallbladder cancer.
\end{abstract}

Keywords: gallbladder cancer; GBC-SD cell; SGC-996 cell; cancer xenograft model; icariin; gemcitabine; NF-kB; cell cycle arrest; apoptosis; synergistic effect

Acta Pharmacologica Sinica (2013) 34: 301-308; doi: 10.1038/aps.2012.162; published online 31 Dec 2012

\section{Introduction}

Gallbladder cancer (GBC) is the fifth most common gastrointestinal cancer worldwide $\mathrm{e}^{[1]}$ and the most aggressive carcinoma of the biliary tract. Although complete surgical resection can cure patients with early stage GBC, approximately $70 \%$ of patients present with inoperable advanced GBC because of lack of early diagnosis ${ }^{[2]}$. Moreover, because of its aggressive biological behavior and resistance to chemotherapy, the prognosis of patients with advanced GBC is associated with an extremely poor survival rate (less than $10 \%)^{[3]}$. For patients with unresectable GBC, treatment options include radiother-

\footnotetext{
* To whom correspondence should be addressed.

E-mail drjianguox@gmail.com (Jian-guo XIA); aroony@gmail.com (Guo-yu CHEN)

Received 2012-08-10 Accepted 2012-10-22
}

apy and systemic chemotherapy. Since the late 1990s, gemcitabine has been considered to be an effective anticancer agent for various cancers. In particular, clinical trials have demonstrated both the efficacy and safety of gemcitabine in patients with GBC. However, the response rate to gemcitabine is not satisfactory, and the overall survival is only approximately 8 months ${ }^{[4]}$. Therefore, novel therapeutic strategies are needed to enhance the response rate and to improve survival.

Icariin, a flavonoid isolated from Epimedi herba, is considered to be the major active ingredient of the traditional Chinese medicine E herba. Icariin is associated with diverse therapeutic effects, including anti-inflammatory effects, antidepressant effects, male sexual function improvement, cardiovascular protection, enhancement of bone healing and neuroprotection ${ }^{[5]}$. In the context of cancer, icariin has been shown to exert an antiproliferative effect on cell growth in 
vitro in mouse Leydig tumor cells, human lung cancer cells, human gastric cancer cells, human leukemia cells, human breast cancer cells and human hepatoma cells ${ }^{[6-10]}$. Although Icariin does not have sufficient potency to be used as a singleagent anticancer drug, these studies suggested that combinatorial therapy with Icariin and conventional chemotherapeutics might provide a treatment option with higher clinical efficacy and better patient survival.

In the present study, our results showed that icariin exhibited antiproliferative and proapoptotic effects on gallbladder cancer cells. We also found that icariin sensitized gallbladder cancer cells to gemcitabine, enhancing gemcitabine-induced growth inhibition and apoptosis. Moreover, we also examined the anticancer activity of icariin in combination with gemcitabine in a murine gallbladder cancer model and found that icariin treatment potentiated the cytotoxicity of gemcitabine in vivo. This synergistic anticancer activity is associated with the downregulation of NF-kB activity and apoptosis-related molecules (Bcl-2, Bcl-xL, and survivin).

\section{Materials and methods Cell culture}

The human gallbladder carcinoma cell lines GBC-SD and SGC996 were obtained from the Shanghai Cell Institute Country Cell Bank. Normal murine gallbladder cells were prepared as previously described ${ }^{[11]}$. All cells were maintained in Dulbecco's modified Eagle's medium (DMEM, Life Sciences, Carlsbad, CA, USA) containing 10\% fetal bovine serum, 100 units/mL penicillin and $100 \mu \mathrm{g} / \mathrm{mL}$ streptomycin. Cells were maintained in a humidified incubator at $37^{\circ} \mathrm{C}$ and $5 \% \mathrm{CO}_{2}$.

\section{Cell growth assay}

Cell proliferation was determined by the 3-(4,5-dimethylthiazol-2-yl)-2,5-diphenyltetrazolium bromide assay (MTT) (Sigma, St Louis, MO, USA), as previously described ${ }^{[12]}$. Briefly, cells were plated at a density of $5 \times 10^{3}$ cells/well in 96-well culture plates. After treatment, $20 \mu \mathrm{L}$ of MTT solution [5 $\mathrm{mg} / \mathrm{mL}$ in phosphate-buffered saline (PBS)] was added to each well and incubated for $2 \mathrm{~h}$. MTT formazan was dissolved in $150 \mu \mathrm{L}$ of isopropanol, and the absorbance was measured at $595 \mathrm{~nm}$ with an ELISA reader (Tecan Group Ltd, Männedorf, Switzerland).

\section{Cell apoptosis analysis}

Cell apoptosis was determined using a FITC Annexin V apoptosis kit (BD Pharmingen, Franklin Lakes, NJ, USA) according to the manufacturer's instructions. In brief, cells were washed with ice-cold PBS and resuspended in binding buffer (10 mmol/L HEPES, pH 7.4, $140 \mathrm{mmol} / \mathrm{L} \mathrm{NaCl}$, and $2.5 \mathrm{mmol} / \mathrm{L} \mathrm{CaCl}_{2}$ ) at a concentration of $1 \times 10^{6}$ cells / $\mathrm{mL}$. Cells were stained with annexin V-FITC and propidium (PI) for $15 \mathrm{~min}$ in the dark before analysis by a flow cytometer (Beckman Coulter Inc, Miami, FL, USA).

\section{Western blot analysis}

Following treatment, cells were washed with ice-cold PBS and harvested in $100 \mu \mathrm{L}$ of cell lysis buffer (Cell Signaling, Danvers, MA, USA) containing protease inhibitors (Sigma, St Louis, MI, USA). Extracted proteins were separated by SDSPAGE and then transferred electrophoretically onto a polyvinylidene difluoride membrane (Millipore, Billerica, MA, USA). Proteins were probed with specific antibodies as previously described $^{[13]}$.

\section{Caspase-3 activity}

Caspase-3 activity was measured in the cell lysate by a colorimetric assay according to the manufacturer's instructions (Keygen Biotech, Nanjing, China). Briefly, $1 \times 10^{6}$ cells were extensively washed with PBS and resuspended in $50 \mu \mathrm{L}$ lysis buffer before incubation in an ice bath for $60 \mathrm{~min}$. The solution was then centrifuged at $10000 \times g$ for 1 minute. The supernatant was collected and incubated with an enzyme-specific colorimetric substrate for $4 \mathrm{~h}$ at $37^{\circ} \mathrm{C}$, and the colorimetric product was measured by an ELISA reader at a wavelength of $405 \mathrm{~nm}$.

\section{Analysis of cell cycle}

The cell cycle was analyzed as previously described ${ }^{[12]}$. Following treatment, cells were collected and fixed with $70 \%$ cold ethanol at $4{ }^{\circ} \mathrm{C}$ overnight. DNA was stained with propidium iodide $(0.05 \mathrm{mg} / \mathrm{mL})$ and RNase $(2 \mathrm{mg} / \mathrm{mL})$ for $30 \mathrm{~min}$ at room temperature. A FACScan flow cytometer (Beckman Coulter Inc, Miami, Florida, USA) was used to analyze cells, and the percentage of cells in the $G_{0} / G_{1}, S$, and $G_{2} / M$ phases of the cell cycle was assessed with Cell Lab Quanta SC Software.

\section{NF-KB p65 activity analysis}

Following treatment, the nuclear extract was prepared using a nuclear extract kit (Active Motif, Carlsbad, CA, USA), and the activity of NF-kB p65 was examined using an ELISA kit (Active Motif, Carlsbad, CA, USA).

\section{In vivo xenograft experiments}

The animal experiments were performed in accordance with CAPN (China Animal Protection Law), and the protocols were approved by the Animal Care and Use Committee of the First Affiliated Hospital, Nanjing Medical University. Female BALB/c $(n u / n u)$ mice, 6 weeks old, purchased from the Animal Centre of China (Beijing, China), were housed with a light/dark cycle of 12/12 h and allowed free access to rodent chow and water. GBC-SD cells were harvested from subconfluent cultures and washed in serum-free medium before being resuspended in PBS. The mice were anesthetized using ethyl ether, and $10^{5}$ cells were injected subcutaneously into the right and left abdominal regions with a gauge needle. Tumor appearance was inspected twice per week. Once tumor masses became established and palpable, animals were randomized into 4 groups ( $n=15$ per group) to receive intraperitoneal injections as follows: (A) vehicle $(0.9 \%$ sodium chloride plus $1 \%$ DMSO) (B) Icariin (40 mg/ kg, dissolved in vehicle) alone, (C) gemcitabine $(125 \mathrm{mg} / \mathrm{kg}$, dissolved in 
vehicle) ${ }^{[14]}$ alone, or (D) Icariin and gemcitabine in combination twice per week for 2 weeks. Tumor volumes and body weight were measured twice per week. Tumor volume was measured along the longest orthogonal axes and calculated as volume $=\left(\right.$ length $\times$ width $\left.{ }^{2}\right) / 2$, where width was the shortest measurement. All mice were sacrificed $35 \mathrm{~d}$ after the first day of treatment.

\section{Statistical analysis}

All data are expressed as the mean \pm SD and represent the results of three separate experiments performed in quadruplicate unless otherwise stated. Student's $t$-test was used to evaluate the difference between two groups, and a one-way ANOVA with a post hoc test was used for comparison among three or more groups. $P<0.05$ was considered to be statistically significant.

\section{Results}

Icariin inhibits cell growth and induces apoptosis in gallbladder cancer cells

To determine whether icariin could inhibit the growth of gallbladder cancer cells, the viability of the cells was assessed using the MTT assay after the cells had been treated with increasing concentrations of icariin for $24 \mathrm{~h}$. As shown in Figure $1 \mathrm{~A}$ and $1 \mathrm{~B}$, icariin inhibited GBC-SD cell growth in a dose-dependent manner. Relative to the control, GBC-SD cell viability was reduced up to $27.1 \% \pm 2.2 \%$ after $160 \mu \mathrm{g} / \mathrm{mL}$ icariin treatment for $24 \mathrm{~h}$. SGC996 cells were less sensitive to icariin than GBC-SD cells, showing a reduced viability of $35.5 \% \pm 3.7 \%$ after $160 \mu \mathrm{g} / \mathrm{mL}$ icariin treatment for $24 \mathrm{~h}$. We also evaluated the ability of icariin to induce apoptosis in GBC-SD and SGC996 cells. As shown in Figure 1A and 1B, icariin effectively induced apoptosis in both cell lines, indicating that the loss of cell viability in response to icariin was in part due to apoptosis. In contrast, even at the maximum dose tested, icariin did not significantly affect the viability of normal mouse gallbladder cells.

\section{Icariin inhibits antiapoptotic molecules in GBC-SD cells}

To explore the mechanism underlying icariin-induced apoptosis, we carried out Western blot analysis on GBC-SD cells. As shown in Figure 2A and 2B, icariin treatment caused a decrease in the level of caspase- 3 and an increase in the PARP level while elevating caspase- 3 activity, further confirming that the antiproliferative activity of icariin was mediated by inducing apoptosis. Our results also revealed that icariin decreased the levels of the antiapoptotic proteins Bcl-2, Bcl-xL, and survivin in a dose-dependent manner, suggesting that icariin induced apoptosis at least partially by inhibiting antiapoptotic molecules.

\section{Icariin inhibits activation of NF-kB}

The NF-kB signaling pathway plays a crucial role in cancer cell survival and is known to regulate the expression of survivin and Bcl family molecules ${ }^{[15]}$. Therefore, we examined NF- $\mathrm{kB}$ activity in GBC-SD cells to investigate the effect of icariin on
NF-KB activity. Icariin treatment led to a dose-dependent decrease in NF-kB activity as shown in Figure 2C, indicating that icariin downregulated antiapoptotic molecules by inhibiting NF-KB activity.

\section{Icariin sensitizes gallbladder cancer cells to gemcitabine}

To explore whether icariin could enhance the chemosensitivity of gallbladder cancer cells to gemcitabine, we examined the effect of treatment with gemcitabine alone or in combination with icariin on the growth of GBC-SD and SGC996 cells. Because icariin at $40 \mu \mathrm{g} / \mathrm{mL}$ had no effect on normal mouse gallbladder cells and significantly inhibited cell growth in both tested cell lines, we used $40 \mu \mathrm{g} / \mathrm{mL}$ icariin in the combinational treatment with gemcitabine. As shown in Figure 3A, treatment with either gemcitabine or icariin alone caused a $42 \%$ and $47 \%$ loss of viable GBC-SD cells, respectively. However, combination treatment with gemcitabine and icariin dramatically reduced the viable GBC-SD cells to $28 \%$. A similar pattern was found in SGC-996 cells when the cells were treated with gemcitabine, icariin or both. To evaluate this synergism, we calculated the combination index value according to Chou's method ${ }^{[16]}$. The results showed that icariin caused a synergistic loss of cell viability when combined with gemcitabine $(\mathrm{CI}=0.694$ and 0.712 for GBC-SD and SGC-996 cells, respectively).

\section{Icariin sensitized GBC-SD and SGC996 cells to apoptosis induced by gemcitabine}

Next, we examined whether the enhanced antiproliferative effect was mediated by apoptosis. Compared with treatment with gemcitabine alone, combination treatment with icariin and gemcitabine induced significantly more apoptosis in both tested gallbladder cancer cell lines, indicating that icariin potentiates the cytotoxic effect of gemcitabine by inducing apoptosis (Figure 3B).

\section{Icariin enhanced gemcitabine-induced apoptosis by regulating} NF-KB and its downstream genes

The mechanism by which icariin sensitized gallbladder cancer cells was investigated using GBC-SD cells. Our results demonstrated that combinational therapy with icariin and gemcitabine caused additional inhibition of apoptosis-related molecules and a marked increase in caspase-3 activity that correlated with a dramatic inhibition in NF-kB activity (Figure 4A, 4B, and 4C). As shown in Figure 4C, icariin inhibited the constitutive action of NF-kB as well as gemcitabine-induced NF- $\mathrm{kB}$ activity. Combining these data with the initial findings, these results suggested that icariin sensitized cells to gemcitabine-induced apoptosis by inhibiting NF-kB activity and causing the ensuing downregulation of apoptosis-related genes.

\section{Icariin potentiated gemcitabine-induced $\mathrm{G}_{0}-\mathrm{G}_{1}$ arrest}

Untreated GBC-SD cells showed a similar cell cycle pattern, with most cells in S-phase and a lower proportion in the $\mathrm{G}_{0}-\mathrm{G}_{1}$ and $G_{2}$ phases. Gemcitabine treatment alone increased $G_{0}-G_{1}$ 

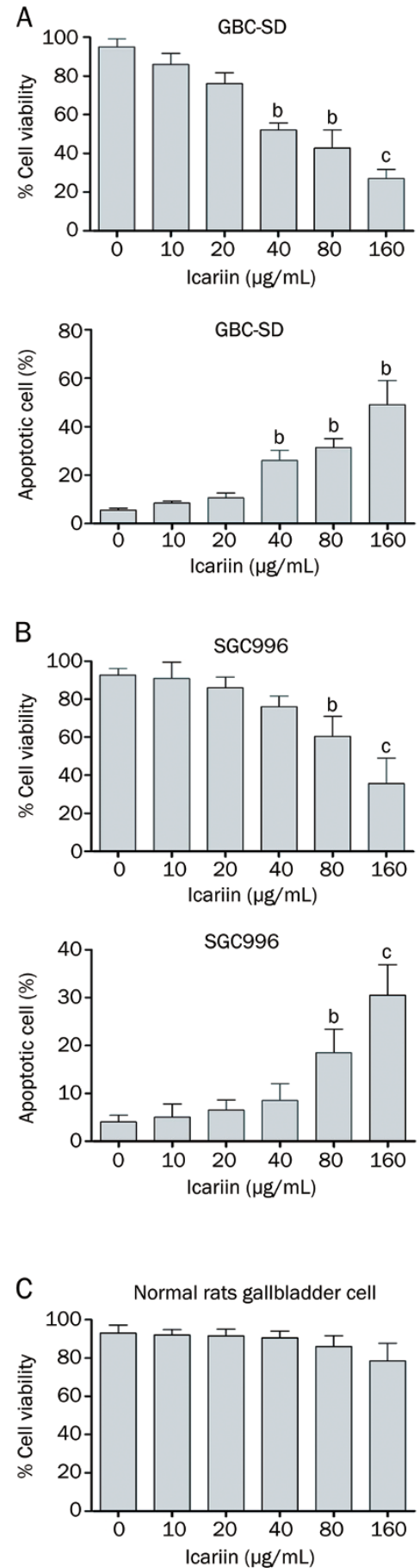
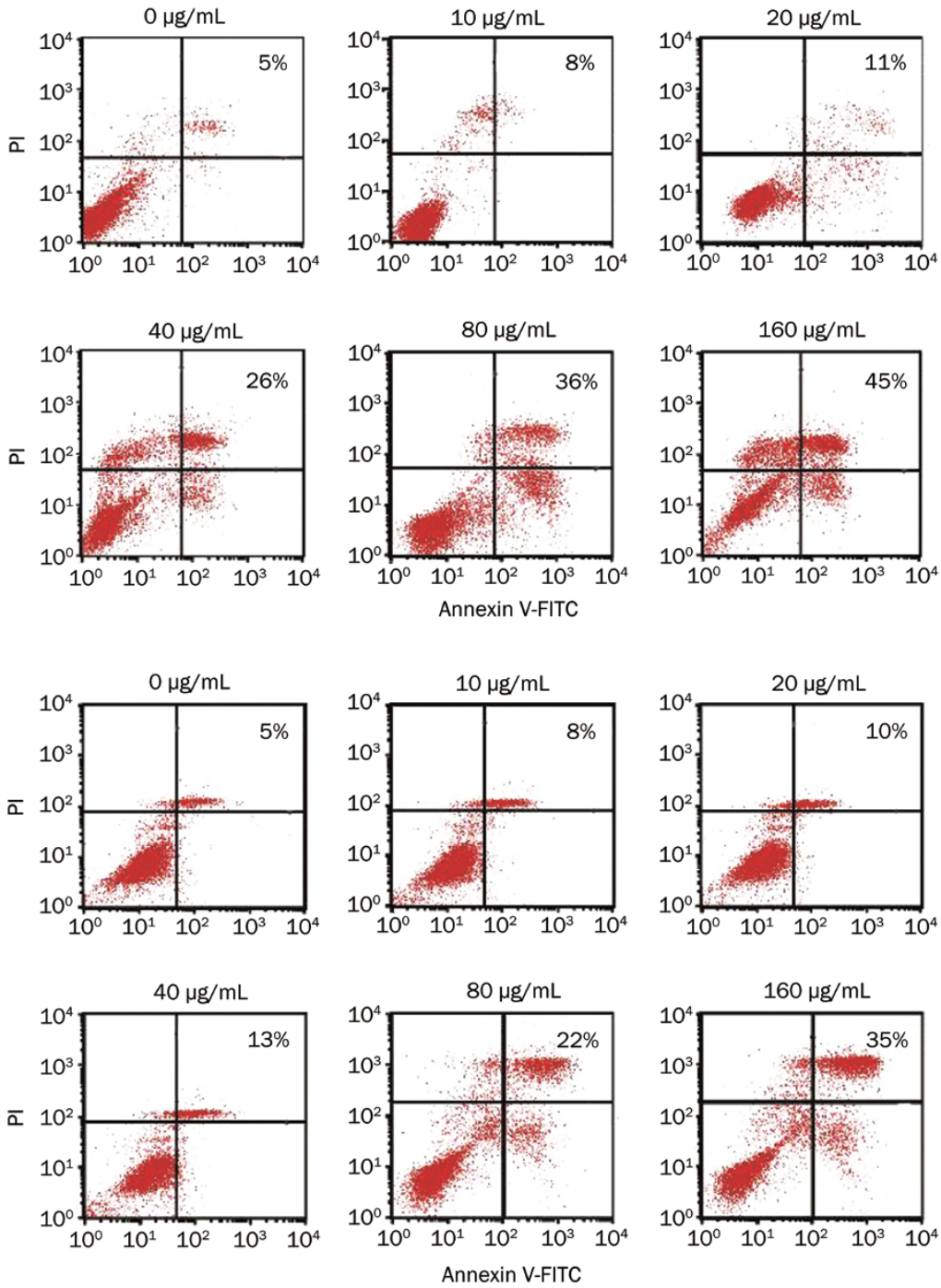

Figure 1. Cell growth inhibition and apoptosis induction by icariin in GBC-SD (A) and SGC-996 (B) cells, and the effect of icariin on normal mouse gallbladder cells (C). Cells were treated with the indicated concentration of Icariin for $24 \mathrm{~h}$. Cell viability was analyzed by the MTT assay, and apoptosis was measured by flow cytometry. Mean \pm SD. $n=3 .{ }^{b} P<0.05,{ }^{c} P<0.01$ vs $0 \mu \mathrm{g} / \mathrm{mL}$ icariin. cell cycle arrest relative to the control as shown in Figure 4D. Although icariin alone did change the cell cycle distribution, icariin and gemcitabine together resulted in an increased cell population arrested at $\mathrm{G}_{0}-\mathrm{G}_{1}$ phase. These results demonstrated that icariin treatment enhanced the cell cycle arrest caused by gemcitabine. Taken together, these in vitro results verified that icariin sensitizes gallbladder cancer cells to gemcitabine therapy and potentiates apoptosis by inhibiting NF-kB activity.
Icariin potentiates the in vivo antitumor effect of gemcitabine in GBC-SD and SGC996 cells

Based on the aforementioned results, we evaluated the in vivo therapeutic effect of icariin and gemcitabine treatment using an in vivo xenograft mouse model. Icariin was administered at $40 \mathrm{mg} / \mathrm{kg}$, and gemcitabine was given at $125 \mathrm{mg} / \mathrm{kg}$. Treatment started when the tumor size reached $0.5 \mathrm{~cm}^{3}$. The efficacy of the treatment was evaluated by measuring the tumor volume during treatment. As shown in Figure 5, treatment with icariin and gemcitabine resulted in significantly smaller 
A

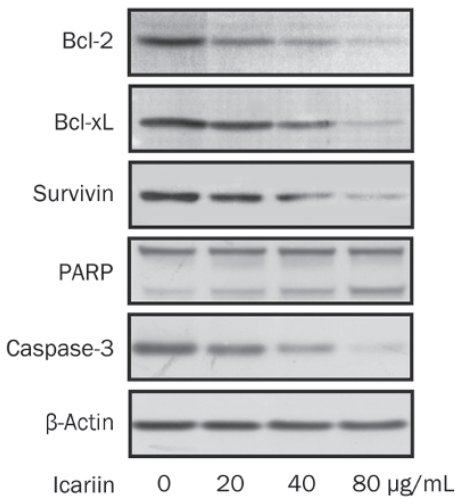

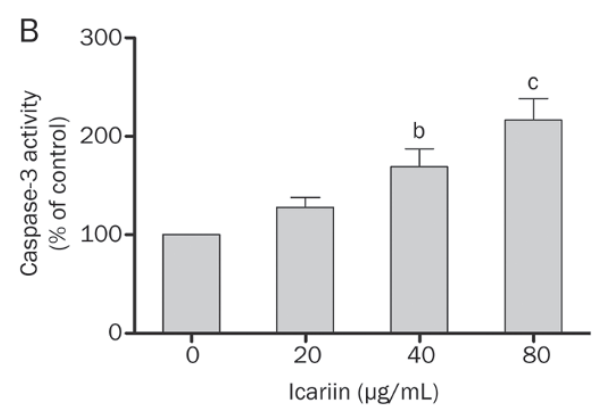

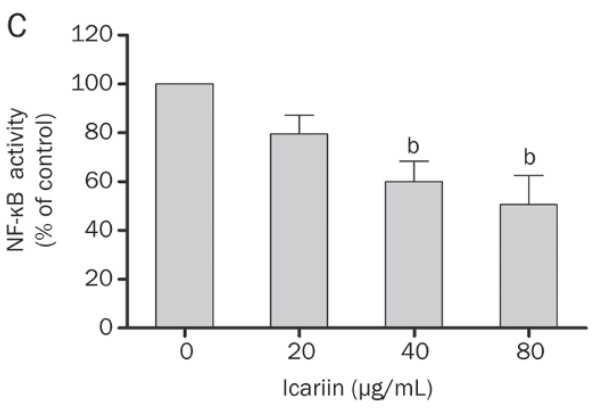

Figure 2. Downregulation of apoptosis-related proteins (A), increased caspase-3 activity (B) and decreased NF-KB activity (C) in GBC-SD cells following icariin treatment. Cells were treated with the indicated concentration of Icariin for $24 \mathrm{~h}$. Expression of apoptosis-related proteins was analyzed by Western blotting; caspase-3 activity was measured by a colorimetric assay; NF-kB activity was determined using an ELISA. Mean \pm SD. ${ }^{b} P<0.05$, ${ }^{\mathrm{c}} P<0.01$ $v s 0 \mu \mathrm{g} / \mathrm{mL}$ icariin.
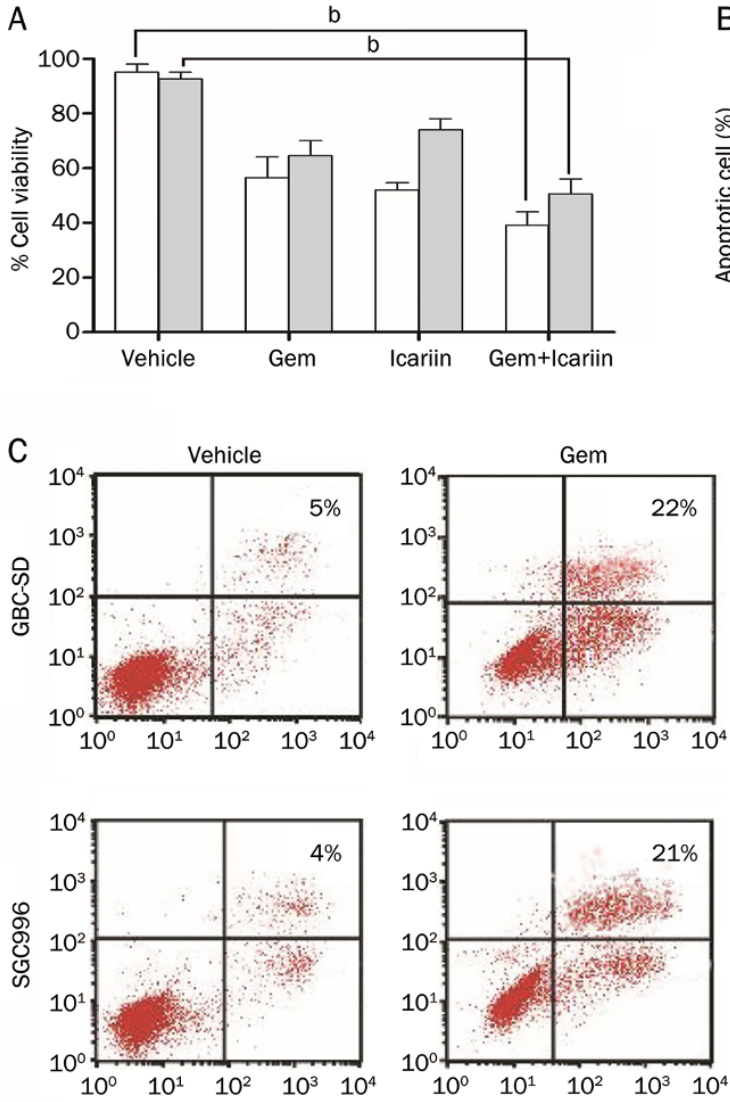
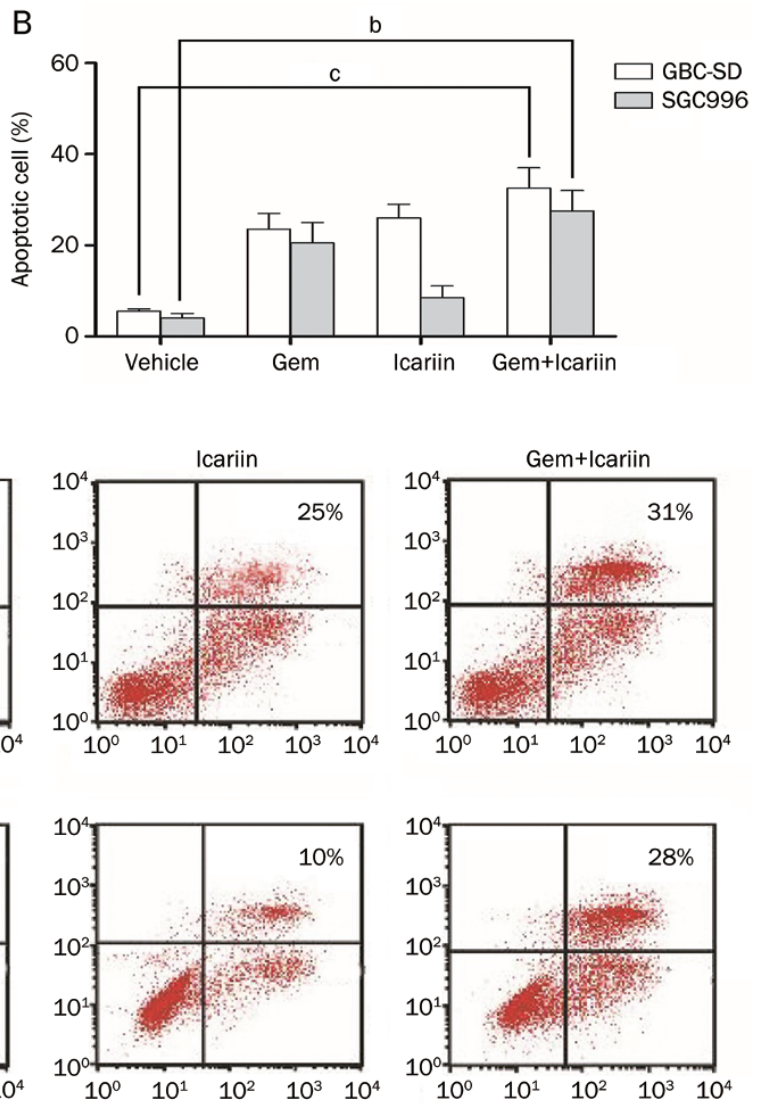

Figure 3. Enhanced anti-proliferative activity (A) and induction of apoptosis (B) of gemcitabine when administered in combination with icariin and representative cytometric apoptosis graphs $(C)$. Cells were treated with both icariin $(40 \mu \mathrm{g} / \mathrm{mL})$ and gemcitabine $(0.5 \mu \mathrm{mol} / \mathrm{L})$ for $24 \mathrm{~h}$. Cell viability was analyzed by the MTT assay, and apoptosis was measured by flow cytometry. Mean \pm SD. $n=3 .{ }^{b} P<0.05,{ }^{c} P<0.01$ vs the vehicle.

tumors than in control mice $(P<0.05)$ or mice treated with gemcitabine or icariin alone $(P<0.05)$.

\section{Discussion}

Owing to its late presentation and aggressive behavior, gall- bladder cancer remains one of the most malignant cancers, with an extremely low 5-year survival rate. In recent years, the actions of natural compounds that exert anti-cancer activity without harming normal cells have been widely investigated in diverse cancer cells. Combination treatment with natural 
A

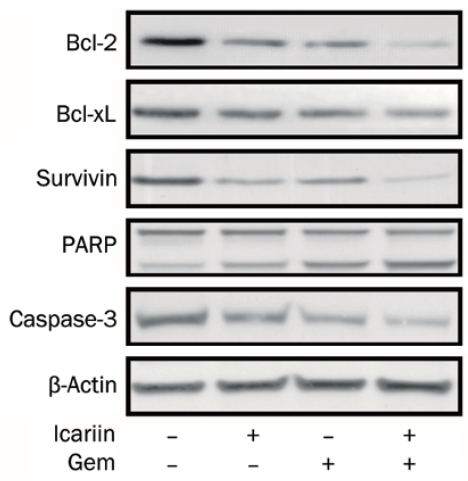

C

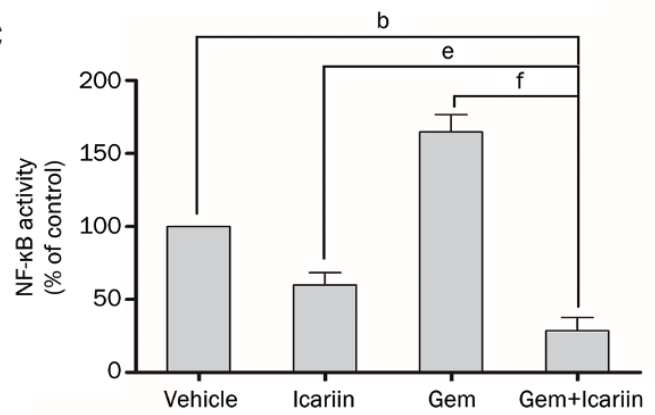

B

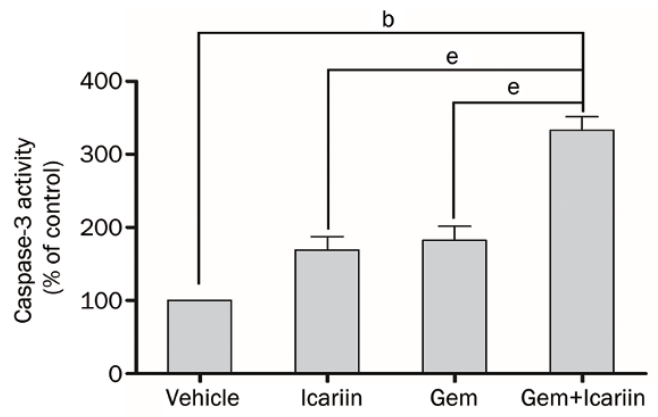

D
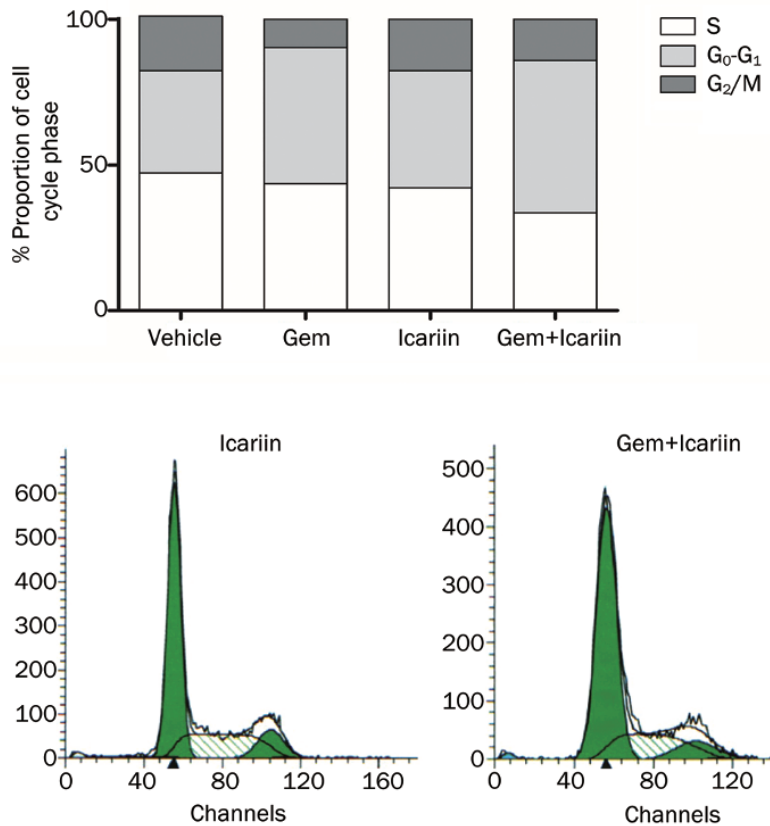

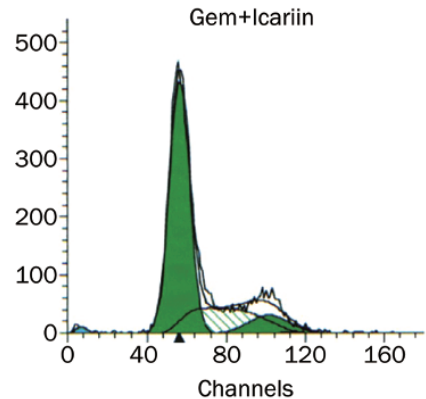

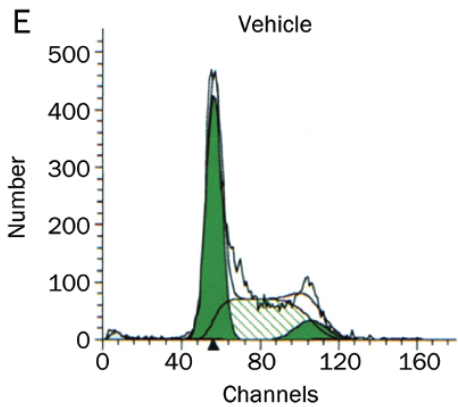

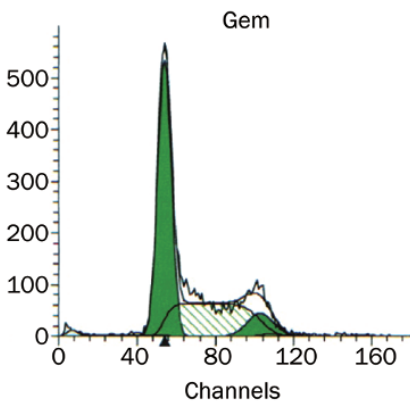

Figure 4. Downregulation of apoptosis-related proteins (A), increased caspase-3 activity (B), decreased NF-kB activity (C), and enhanced $\mathrm{G}_{0}-\mathrm{G}_{1}$ cell cycle arrest (D) in GBC-SD cells treated with both gemcitabine and Icariin with representative cell cycle analysis graphs (E). GBC-SD cells were treated with both icariin $(40 \mu \mathrm{g} / \mathrm{mL})$ and gemcitabine $(0.5 \mu \mathrm{mol} / \mathrm{L})$ for $24 \mathrm{~h}$. Expression of apoptosis-related proteins was analyzed by Western blotting; caspase-3 activity was measured by a colorimetric assay; NF-KB activity was determined using an ELISA. Cell cycle distribution is presented as the mean. Mean \pm SD. $n=3 .{ }^{b} P<0.05$ vs the vehicle; ${ }^{e} P<0.05,{ }^{f} P<0.01$ vs the treatment with icariin or gemcitabine alone.

compounds has also received considerable attention because the synergistic interaction of several agents might decrease the systemic toxicity of chemotherapy by achieving the same efficacy with lower doses. However, only one natural compound has been reported to be able to potentiate the cytotoxic activity of platinum anticancer drugs in gallbladder cancer cells ${ }^{[17]}$. In this study, our results showed that icariin, by inhibiting NF-KB activity, exhibited antitumor activity and potentiated the antitumor activity of gemcitabine in gallbladder cancer.

It has been reported that icariin can regulate NF-kB activity in a variety of cell types ${ }^{[5]}$. Xu et al reported that icariin enhanced endothelial nitric oxide synthase expression in human endothelial cells and that this was correlated with an increase in NF-KB activity ${ }^{[18]}$. Icariin has also been reported to promote cardiac differentiation of mouse embryonic stem cells by activating NF-kB ${ }^{[19]}$. By contrast, icariin attenuated lipopolysaccharide-induced neuroinflammation, lung inflammation, osteoclast differentiation and bone resorption by suppressing NF- $\mathrm{KB}^{[20-23]}$. In the present study, we found that the inhibition of NF-KB activity by icariin led to apoptosis in gallbladder cancer cells, which have highly active NF- $\mathrm{KB}^{[23]}$. It seemed that the regulatory effect of icariin was cell specific and that icariin might only suppress NF- $\mathrm{KB}$ activity in cells with abnormally high NF-KB activity such as tumor cells or cells stimulated by external stimuli.

Emerging evidence has shown that NF-kB plays a crucial role in cell proliferation, invasion, apoptosis inhibition, chemoresistance and radioresistance ${ }^{[24]}$. Activation of NF-kB by several conventional chemotherapeutics has also resulted in unfavorable clinical outcomes. A number of studies have 
A

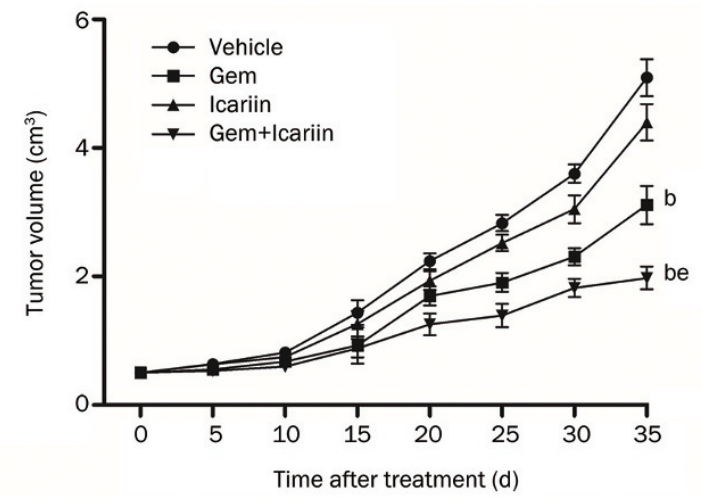

B
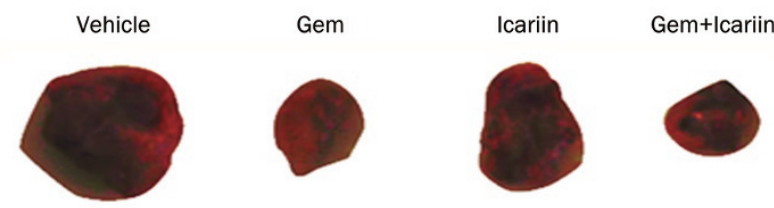

Figure 5. Measurements of gallbladder tumor volume at the indicated time points, depicting the in vivo therapeutic efficacy of icariin and gemcitabine $(A)$ with a representative picture of a tumor at the end of the experiment (B). $n=15 .{ }^{b} P<0.05$ vs animals treated with vehicle. ${ }^{\mathrm{e}} P<0.05$ vs animals treated with icariin or gemcitabine alone.

shown that the antitumor effect of gemcitabine was enhanced by inhibiting NF-KB. In pancreatic cancer cells, the efficacy of gemcitabine was found to be augmented when NF-kB activity was inhibited by escin, emodin, gum mastic, resveratrol or thymoquinone ${ }^{[24-28]}$. In addition to this finding for pancreatic cancer, enhanced chemosensitivity to gemcitabine has also been reported to be associated with decreased NF-kB activity in osteosarcoma, colon cancer, breast cancer, bladder cancer and non-small cell lung cancer cells ${ }^{[15,29-31]}$. In agreement with these studies, we found that the sensitization of gallbladder cancer cells to gemcitabine by icariin correlated with suppressed NF-kB activity. Taken together, these results indicate that Therefore, the regulation of NF-KB activity could be a possible new treatment for the chemosensitization of human gallbladder cancer to gemcitabine. Furthermore, Wu et al also suggested that active NF-kB contributes to the highly invasive and metastatic behavior of gallbladder cancer ${ }^{[23]}$. Therefore, by suppressing NF-KB activity, icariin might be able to inhibit cell invasion, in addition to inducing apoptosis and suppressing cell growth.

To further understand how icariin sensitizes cells to gemcitabine, we examined cell cycle progression and the expression of the downstream genes of NF-KB. We found that icariin downregulated the constitutive expression of the apoptosisrelated molecules Bcl-2, Bcl-xL, and survivin, and combination treatment showed a greater suppressive effect on these molecules. It has been suggested that survivin prevents the cell cycle through $\mathrm{M}$ phase and induces spontaneous apoptosis ${ }^{[25]}$. It is conceivable that the inhibition of survivin by icariin also contributes to the enhanced cytotoxicity of gemcitabine. In summary, our results indicated that icariin plays a synergistic role in gemcitabine-induced apoptosis and growth inhibition in gallbladder cancer cells.

To examine whether the enhanced cell growth inhibition and induction of apoptosis could be recapitulated in vivo, we treated a gallbladder cancer mouse model with icariin, gemcitabine or a combination of icariin and gemcitabine. Our results showed that the combination treatment led to a significant inhibition of tumor growth compared to treatment with either agent alone. More importantly, as shown in Figure 1C, icariin had a minimal effect on the viability of normal cells, so the combination of gemcitabine with icariin could achieve a greater therapeutic effect without causing systemic toxicity, a beneficial characteristic of combination therapy with two or more chemotherapeutics.

In conclusion, our results demonstrated that icariin can potentiate the antitumor activity of gemcitabine on human gallbladder cancer cells both in vitro and in vivo. The underlying mechanisms may be, at least in part, due to icariin-induced suppression of NF-kB and NF-kB-regulated gene products. Given the low toxicity of icariin to normal tissue and our present findings, we believe that icariin may also synergize with gemcitabine against other cancers such as pancreatic cancer. However, further studies, including preclinical studies and clinical trials, are needed to support our strategy for the treatment for gallbladder cancer.

\section{Acknowledgements}

This work was supported by grants from National Natural Science Foundation of China ( № 30901443) and from the Opening Project of Medical Key Disciplines in Jiangsu Province (№ XK03 200904).

\section{Author contribution}

Dian-cai ZHANG, Jian-guo XIA, and Guo-yu CHEN designed research; Dian-cai ZHANG and Jin-long LIU performed research; Dian-cai ZHANG and Yong-bin DING analyzed data; Dian-cai ZHANG and Jian-guo XIA wrote the paper.

\section{References}

1 Reid KM, Ramos-De la Medina A, Donohue JH. Diagnosis and surgical management of gallbladder cancer: a review. J Gastrointest Surg 2007; 11: 671-81.

2 Kaneoka Y, Yamaguchi A, Isogai M, Harada T, Suzuki M. Hepatoduodenal ligament invasion by gallbladder carcinoma: histologic patterns and surgical recommendation. World J Surg 2003; 27: 260-5.

3 Wang JW, Peng SY, Li JT, Wang Y, Zhang ZP, Cheng Y, et al. Identification of metastasis-associated proteins involved in gallbladder carcinoma metastasis by proteomic analysis and functional exploration of chloride intracellular channel 1. Cancer Lett 2009; 281 : 71-81.

4 Zhu AX, Hong TS, Hezel AF, Kooby DA. Current management of gallbladder carcinoma. Oncologist 2010; 15: 168-81.

5 Chen Y, Huang JH, Ning Y, Shen ZY. Icariin and its pharmaceutical efficacy: research progress of molecular mechanism. Zhong Xi Yi Jie He Xue Bao 2011; 9: 1179-84. 
6 Huang X, Zhu D, Lou Y. A novel anticancer agent, icaritin, induced cell growth inhibition, G1 arrest and mitochondrial transmembrane potential drop in human prostate carcinoma PC-3 cells. Eur J Pharmacol 2007; 564: 26-36.

7 Li S, Dong P, Wang J, Zhang J, Gu J, Wu X, et al. Icariin, a natural flavonol glycoside, induces apoptosis in human hepatoma SMMC7721 cells via a ROS/JNK-dependent mitochondrial pathway. Cancer Lett 2010; 298: 222-30.

8 Lin CC, Ng LT, Hsu FF, Shieh DE, Chiang LC. Cytotoxic effects of Coptis chinensis and Epimedium sagittatum extracts and their major constituents (berberine, coptisine and icariin) on hepatoma and leukaemia cell growth. Clin Exp Pharmacol Physiol 2004; 31: 65-9.

9 Wang $\mathrm{Y}$, Dong $\mathrm{H}$, Zhu M, Ou Y, Zhang J, Luo $\mathrm{H}$, et al. Icariin exterts negative effects on human gastric cancer cell invasion and migration by vasodilator-stimulated phosphoprotein via Rac1 pathway. Eur J Pharmacol 2010; 635: 40-8.

10 Wang Q, Hao J, Pu J, Zhao L, Lu Z, Hu J, et al. Icariin induces apoptosis in mouse MLTC-10 Leydig tumor cells through activation of the mitochondrial pathway and down-regulation of the expression of piwil4. Int J Oncol 2011; 39: 973-80.

11 Coad RA, Dutton JR, Tosh D, Slack JM. Inhibition of Hes1 activity in gall bladder epithelial cells promotes insulin expression and glucose responsiveness. Biochem Cell Biol 2009; 87: 975-87.

12 Cheng H, An SJ, Zhang XC, Dong S, Zhang YF, Chen ZH, et al. In vitro sequence-dependent synergism between paclitaxel and gefitinib in human lung cancer cell lines. Cancer Chemother Pharmacol 2011; 67: $637-46$.

13 Huang H, Chen D, Li S, Li X, Liu N, Lu X, et al. Gambogic acid enhances proteasome inhibitor-induced anticancer activity. Cancer Lett 2011; 301: 221-8.

14 Mita Y, Ajiki T, Kamigaki T, Okazaki T, Hori H, Horiuchi $\mathrm{H}$, et al. Antitumor effect of gemcitabine on orthotopically inoculated human gallbladder cancer cells in nude mice. Ann Surg Oncol 2007; 14 : 1374-80.

15 Guo X, Xu B, Pandey S, GoessI E, Brown J, Armesilla AL, et al. Disulfiram/copper complex inhibiting NFkappaB activity and potentiating cytotoxic effect of gemcitabine on colon and breast cancer cell lines. Cancer Lett 2010; 290: 104-13.

16 Chou TC, Talalay P. Quantitative analysis of dose-effect relationships: the combined effects of multiple drugs or enzyme inhibitors. Adv Enzyme Regul 1984; 22: 27-55.

17 Wang W, Sun YP, Huang XZ, He M, Chen YY, Shi GY, et al. Emodin enhances sensitivity of gallbladder cancer cells to platinum drugs via glutathion depletion and MRP1 downregulation. Biochem Pharmacol 2010; 79: 1134-40.

$18 \mathrm{Xu} \mathrm{HB}$, Huang ZQ. Icariin enhances endothelial nitric-oxide synthase expression on human endothelial cells in vitro. Vascul Pharmacol 2007; 47: 18-24.
19 Wo Y, Zhu D, Yu Y, Lou Y. Involvement of NF-kappaB and AP-1 activation in icariin promoted cardiac differentiation of mouse embryonic stem cells. Eur J Pharmacol 2008; 586: 59-66.

20 Hsieh TP, Sheu SY, Sun JS, Chen MH. Icariin inhibits osteoclast differentiation and bone resorption by suppression of MAPKs/NFkappaB regulated HIF-1alpha and PGE(2) synthesis. Phytomedicine 2011; 18: 176-85.

21 Xu CQ, Liu BJ, Wu JF, Xu YC, Duan XH, Cao YX, et al. Icariin attenuates LPS-induced acute inflammatory responses: involvement of PI3K/ Akt and NF-kappaB signaling pathway. Eur J Pharmacol 2010; 642: 146-53.

22 Zeng KW, Fu H, Liu GX, Wang XM. Icariin attenuates lipopolysaccharide-induced microglial activation and resultant death of neurons by inhibiting TAK1/IKK/NF-kappaB and JNK/p38 MAPK pathways. Int Immunopharmacol 2010; 10: 668-78.

$23 \mathrm{Wu}$ W, Pan C, Yu H, Gong H, Wang Y. Heparanase expression in gallbladder carcinoma and its correlation to prognosis. J Gastroenterol Hepatol 2008; 23: 491-7.

24 Wang YW, Wang SJ, Zhou YN, Pan SH, Sun B. Escin augments the efficacy of gemcitabine through down-regulation of nuclear factorkappaB and nuclear factor-kappaB-regulated gene products in pancreatic cancer both in vitro and in vivo. J Cancer Res Clin Oncol 2012; 138: 785-97.

25 Banerjee S, Kaseb AO, Wang Z, Kong D, Mohammad M, Padhye S, et al. Antitumor activity of gemcitabine and oxaliplatin is augmented by thymoquinone in pancreatic cancer. Cancer Res 2009; 69: 5575-83.

26 Harikumar KB, Kunnumakkara AB, Sethi G, Diagaradjane P, Anand P, Pandey MK, et al. Resveratrol, a multitargeted agent, can enhance antitumor activity of gemcitabine in vitro and in orthotopic mouse model of human pancreatic cancer. Int J Cancer 2010; 127: 257-68.

27 Huang XY, Wang HC, Yuan Z, Li A, He ML, Ai KX, et al. Gemcitabine combined with gum mastic causes potent growth inhibition and apoptosis of pancreatic cancer cells. Acta Pharmacol Sin 2010; 31: 741-5.

28 Liu A, Chen H, Tong H, Ye S, Qiu M, Wang Z, et al. Emodin potentiates the antitumor effects of gemcitabine in pancreatic cancer cells via inhibition of nuclear factor-kappaB. Mol Med Report 2011; 4: 221-7.

29 Kamat AM, Sethi G, Aggarwal BB. Curcumin potentiates the apoptotic effects of chemotherapeutic agents and cytokines through downregulation of nuclear factor-kappaB and nuclear factor-kappaBregulated gene products in IFN-alpha-sensitive and IFN-alpha-resistant human bladder cancer cells. Mol Cancer Ther 2007; 6: 1022-30.

30 Rundall BK, Denlinger CE, Jones DR. Suberoylanilide hydroxamic acid combined with gemcitabine enhances apoptosis in non-small cell lung cancer. Surgery 2005; 138: 360-7.

31 Zhang B, Shi ZL, Liu B, Yan XB, Feng J, Tao HM. Enhanced anticancer effect of gemcitabine by genistein in osteosarcoma: the role of Akt and nuclear factor-kappaB. Anticancer Drugs 2010; 21: 288-96. 